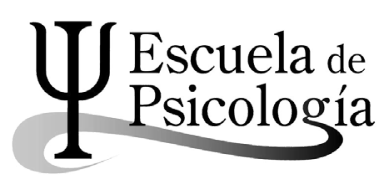

Wímb lu, Rev. electrónica de estudiantes Esc. de psicología, Univ. de Costa Rica. 9(1): 67-81, 2014 / ISSN: 1659-2107

\title{
ESPACIO PÚBLICO URBANO COMO CATALIZADOR DE ACTIVIDAD FÍSICA Y BIENESTAR PSICOLÓGICO
}

Public urban space as motor of physical activity and psychological well-being

Yocelyn Urbina Casasola*

Resumen: El presente artículo tiene como objetivo principal, discutir con base a la revisión de literatura los posibles efectos positivos que pueden tener el empleo del espacio público urbano, específicamente las ciclovías sobre el bienestar físico y psicológico de sus usuarios y usuarias. Dado a la evidencia, se halla que dichos espacios inciden sobre los niveles de estrés, calidad de vida, capital social y cohesión social; colaborando en la percepción y desarrollo de ciudades más saludables.

Palabras clave: espacio público urbano, ciclovía, bienestar psicológico, estrés, calidad de vida.

Abstract: This article is aimed, to discuss based on the review of literature the possible positive effects that can have the use of urban public space, specifically the bike lanes on the physical and psychological well-being of its users. Given the evidence, is that such spaces have an impact on the levels of stress, quality of life, social capital and social cohesion; collaborating in the perception and development of healthier cities.

Key Words: space urban public, bicycle path, psycholigical well-being, stress, quality of life.

Estudiante de licenciatura en psicología. Universidad de Costa Rica. Correo electrónico: yosscycasu@gmail.com, yocelyn.urbina.c@outlook.com.

Recepción: 17/10/2013 Aceptación: 6/02/2014 


\section{Introducción}

Los espacios públicos urbanos son áreas potenciales y de intercambio social, que además de permitir el tránsito y movilización de las personas permiten el desarrollo de interacciones entre sus habitantes y la ciudad misma, lo que conlleva a que se generen significados e interpretaciones sobre dichos espacios. Estos, al ser un producto concreto de diversas y sucesivas intenciones, generan una historia que es acumulada y reinterpretada, por ende cuenta con cierto grado de significado dentro de la vida individual y grupal.

Características como son la cohesión social, seguridad percibida y capital social pueden ser observadas y promocionadas desde diferentes espacios públicos, entre ellos las ciclovías urbanas. Tuesca (2005) ha señalado que las ciclovías como espacio público de construcción social pueden incentivar que sus usuarios(as) interactúen con su ciudad de manera más íntima, aumentando la cohesión social y el capital humano. Además de estimular el uso de la bicicleta como medio de desplazamiento dentro de la ciudad, lo cual conlleva al desarrollo de actividad física.

Diversos estudios han encontrado que la actividad física puede ser un catalizador de bienestar psicológico y social, teniendo efectos positivos sobre la experiencia subjetiva de estrés y ansiedad (Mutrie \& Faulkner, 2004 citado en Jimenez, Miró, Martínez \& Sánchez, 2008). También se ha descrito que el pedalear en bicicleta diariamente ayuda a mejorar la condición física, reduce el riesgo de sobrepeso, fortifica la estructura muscular, disminuye el estrés y ansiedad, además de brindar tiempo de esparcimiento (Rodríguez, 2004 citado en Luci, 2009). En Costa Rica, se han construido ciclovías en algunos sitios como son Esparza, El Roble de Puntarenas, Limón, Hatillo y Cartago pero no se han desarrollados estudios que permitan valorar los efectos que pueden tener estos espacios sobre los niveles de estrés e índices de calidad de vida entre sus usuarios(as).

Las investigaciones referidas al tema de actividad física y efectos psicológicos en los últimos años han sido de ascendente interés pero se carecen de estudios que vinculen dicha asociación con el uso del espacio público, específicamente, las ciclovías. Por lo tanto, no se han podido señalar correlaciones o incluso relaciones causales entre variables psicológicas, actividad física y uso del espacio público. Por tales motivos el presente artículo, aborda el tema de las ciclovías con el objetivo de evaluar el impacto que estas pueden tener sobre las personas en términos de actividad física y bienestar psicológico; indicándose a su vez que para trabajar sobre esta última dimensión, el estudio se centró en las variables de estrés y calidad de vida.

En cuanto a la presentación del documento. Primeramente se aborda una serie de antecedentes investigativos y definiciones referidas a los temas de actividad física-bienestar psicológica, uso de ciclovías y niveles de estrés- calidad 
de vida. Seguidamente se detallan las principales discusiones y conclusiones derivadas de la recopilación de literatura.

\section{Marco Referencial}

\section{Espacio Urbano Público}

El espacio urbano público es un producto concreto de diversas y sucesivas intenciones, generador de una historia acumulada y reinterpretada, cual incide en cierta medida dentro de la vida grupal e individual de sus ciudadanos y ciudadanas. Por medio de los lugares las personas pueden llevar a cabo sus roles culturales y sociales, ya que los lugares en sí son el escenario de la vida pública (Low, 2000).

Según Ferrer (2009) el espacio público en sí es un sitio de tolerancia y convivencia entre nos-otros que permite la presencia de diferencias y conflictos entre sus partes, siendo un lugar de descubrimiento y encuentro para con sus ciudadanos y ciudadanas; generador de sentido con respecto a las prácticas desarrolladas dentro de la ciudad, además de contar con un correlato de índole urbana directamente.

Investigadores como Jacobs (1992) señalan que las ciudades saludables deben brindar distintos usos apoyados entre sí, mencionando que las ciudades deben ser evaluadas no solo en términos de movilidad sino también en cuanto a accesibilidad e interrelación se refiere, debiéndose observar, si la ciudadanía logra moverse fácilmente, con bajo costos económicos y si pueden tener contacto con diferentes espacios, bridando dichas características esbozos sobre la calidad y usos que se le da al espacio urbano.

En cuanto a la adquisición de ciudadanía, el espacio público al cumplir con funciones de estructuración urbana, política y sociocultural, brinda una de las principales condiciones que forman parte de este proceso de adquisición. Desde el espacio urbano se logra crear politización del mundo y de la sociedad; lo cual hace surgir y movilizar el sentido común, mismo que forma parte de las líneas de estructuración del ser y que conllevan a la representación de ciudadanía (Borja, 2002 citado en Ferrer, 2009).

En la actualidad los espacios públicos urbanos han sido invisibilizados, elitizados y privatizados, dándosele a nivel mediático y representativo un mayor valor tanto al transporte privado como a las zonas privadas, situación que ha influido en el incremento de la adquisición de vehículos, acortando las posibilidades de interacción entre ciudadanos y ciudadanas; haciendo que la ciudad sea vista como una zona meramente de paso y no de construcción ciudadana.

Se convierte entonces la supervivencia cultural y social, uno de los mayores retos de las y los habitantes de las ciudades contemporáneas, siendo una 
necesidad la promoción de espacios agradables que permitan el desarrollo de oportunidades de interacciones interpersonales y sociales. Espacios cuales al permitir la democratización de los territorios, incentiven que se dé un mayor uso colectivo, donde la presencia de las personas en dichos espacios recree un mayor sentir de pertenecía y reduzca los índices de criminalidad, ya que el habitar de manera activa las áreas públicas aumenta la percepción de seguridad sobre la ciudad (Jacobs, 1992).

Las medidas empleadas en Costa Rica para descongestionar los centros urbanos como es la restricción vehicular, lo único que ha hecho es aumentar la adquisición de vehículos automotores ya que se ha creado una mayor oferta de espacios para los automóviles. Autores menciona que las ciudades que priorizan el empleo de automóviles propician un aumento en los niveles de inactividad física y sobrepeso (Parra, Goméz, Pratt, et als, 2007 citado en Goméz, Mosquera \& Jacoby, 2009). No obstante, se considera que aquellas ciudades que implementen sistemas de transporte que permitan construir un vínculo funcional con las distintas formas de transporte activo como son el uso de la bicicleta y caminar puede impactar de manera positiva en la promoción de la actividad física y reducción del riesgo de sobrepeso en sus habitantes (Agence de la sante et des services sociax de Montreal, 2006 citado en Gómez et al., 2009).

Acorde al informe mundial de asentamiento humano 2013 sobre planificación y diseño de una movilidad urbana sostenible del Programa de las Naciones Unidas para los Asentamientos Humanos, ONU- Habitat ( 2013) indica que el mayor reto de la movilidad urbana en el presente es pasar del enfoque implementado varias décadas atrás centrado en aumentar la expansión de infraestructura de transporte al enfoque de accesibilidad, orientación que busca que la construcción del espacio urbano brinde mayor optimización de la densidad urbana y sentido de pertenencia por medio de la promoción del uso de transporte no motorizado y mejora de las economías de aglomeración. Desde el enfoque de la accesibilidad emerge el concepto de movilidad urbana asequible, el cual indica que el transporte debe estar al alcance de la mayor parte de la población de la ciudad, en especial para aquellas poblaciones vulnerables que no cuentan con otra forma servicios, bienes y actividades básicas, ya que una movilidad limitada se convierte en un objeto de exclusión social, por ello dicho Programa de las Naciones Unidas propone la importancia de apoyar el transporte urbano no motorizado, mejora en la accesibilidad de los espacios públicos y reducción en los costes de los servicios de transporte como estrategias urbanas y locales para el alcance de los objetivos de la constitución de una movilidad urbana asequible y ciudades accesibles (ONUHabitat, 2013).

Las carreteras son consideradas como "no lugares", entendiéndose como "no lugar", aquel sitio que debido a su diseño estándar y aislado del entorno brindan uniformidad sobre las comunidades (Auge, 2005). El "no lugar" es la negación 
del lugar, diseñado según indicaciones de la época, en donde los sujetos que lo visitan no se detienen ni participan en la dotación de su significado (Auge, 1994 citado en Castrogiovanni, 2007). Un lugar, en cambio es un espacio que puede ser reconocido, con identidad propia y apropiado por medio de las relaciones interpersonales y comunicacionales (Santos, 1994 citado en Castrogiovanni, 2007). Espacios públicos como son las ciclovías y los parques pueden ser considerados como lugares, ya que a través de las actividades que promueven invita al desarrollo de acciones como son interactuar, vincular y compartir entre habitantes, lo cual favorece el proceso de significación.

Las ciclovías, son conceptualizadas como un espacio público diseñado idealmente para el tránsito seguro de los(as) usuarios(as) de bicicletas que conecta zonas residenciales con estaciones y áreas comerciales, cumpliendo funciones de movilización recreación y salud (ARQHYS, 2013). Al ser las ciclovías en principio un espacio excluyente (dirigido a los (as) ciclistas) son percibidas como un espacio de poder y conflicto, las cuáles deben ser compartidas por diferentes clases de usuarios y usuarias que van desde las personas que andan en bicicletas, quienes caminan por ellas, quienes pasean a sus mascotas entre otros (Gomescásseres, 2003).

Diseños y construcciones urbanas como son las ciclovías suscitan la reducción de la dependencia de vehículos motorizados por el uso de movilidades urbanas más sanas que influyen a que se perpetué un mayor sentir de habitar una ciudad más accesible para su población en general y no solo para los (as) dueños(as) de vehículos automotores privados.

\section{Actividad Física y Bienestar Psicológico.}

Se define como actividad física, cualquier movimiento procedente de la contracción muscular esquelética (Escobar, 2003). Dicha actividad, ayuda a distraer a la persona de estresores y estímulos que producen ansiedad, y deriva beneficios como son cambios asertivos en las autopercepciones y autoconfianza, liberación de tensión, generación de cambios positivos en estados ansiosos y depresivos, incentivación de destrezas para el enfrentamiento del estrés y de la vida cotidiana, estimulación de la autoestima y claridad mental (Pate, et al., 1995 citado en Castro 2005).

Por otra parte, el bienestar psicológico también llamado bienestar subjetivo hace referencia a los índices de felicidad personal y satisfacción con la vida; sobre su vinculación con la actividad física se ha encontrado que esta contribuye a mejorar los niveles de salud y calidad de vida, incremento en la esperanza de vida y descenso en las tasas de mortalidad (Marquéz, 1995).

Diferentes estudios señalan que existe una relación entre actividad física y bienestar psicológico. Mencionándose entre ellos el llevado a cabo por un grupo de 
investigadores españoles, quienes trabajaron con una muestra de 208 estudiantes de la Universidad de Averio en Portugal y 239 estudiantes de la Universidad de Granada en España con el propósito de estudiar la asociación entre la práctica de ejercicio físico, hábitos de vida saludable y bienestar psicológico (Jimenez et al., 2008). Entre sus resultados se indica que las personas que practican algún tipo de ejercicio se perciben sobre sí mismas como individuos más saludables, con mejor estado de ánimo (menos tristes, mayor vigor y menos fatiga) y menores índices de estrés tanto en la muestra portuguesa como española (Jimenez et al., 2008). Dichos resultados concuerdan con los expuestos por estudios anteriores que señalan que el ejercicio físico ayuda a mejorar la salud subjetiva, reduce los niveles de ansiedad y depresión clínica, tanto en poblaciones clínicas como no clínicas (Biddle, Fox \& Boutchener, 2000; Akandere \& Tekin, 2005; Lawlor \& Hopker, 2001 citado en Jimenez et al., 2008).

Se ha indicado que para obtener beneficios subjetivos asociados a la actividad física es importante que dicha actividad sea moderada - baja, lo cual se representa entre un rango de 30-60\% de distinción entre la frecuencia cardiaca en estado máximo y de reposo, con un tiempo de 20-30 minutos de duración (Devenes, 1981, citado en Marquéz, 1995).

De acuerdo con Urzúa 2010 a través de investigaciones psiconeuroinmunoendocrinólogica se ha logrado ir encontrando resultados que permitan sentar las bases de la relación inmunidad y estado de ánimo, tanto en poblaciones clínicas como en población general. Una investigación llevada a cabo con una muestra de estudiantes universitarios(as) en Perú que tuvo la finalidad de evaluar las relaciones entre estrés, salud mental y cambios inmunológicos reporta que el estrés académico debido a los exámenes provocan un aumento en la producción de superóxido por neutrófilos y variaciones en el número de células NK ( Guarino, Gavida, Antor \& Caballero, 2000). Además de señalar que es posible que el efecto de somatización e intensidad experimentada y percibida del estrés asociado se pueda deber de forma parcial a la disminución de la actividad física (Guarino et al., 2000).

Se hallado que la actividad física trae beneficios tanto a nivel individual como social, ya que aquellas comunidades que integren la actividad física como una normativa social tienden hacer más sanas y disponer de mayores índices de capital humano (Mutrie \& Faulkner, 2001 citado en Jimenez et al., 2008). Pudiendo entrar aquí en juego las ciclovías, parques, plazas y entre otros espacios públicos construidos, cuales tienen entre sus objetivos el promover el ejercicio físico.

Con respecto al eje de las ciclovías, se ha encontrado que el esfuerzo físico diario desarrollado a la hora de pedalear al andar en bicicleta no sólo permite combatir enfermedades cardiovasculares e hipertensión ( Saraba, et als, 2005 citado en Luci, 2009) sino que también reduce el riesgo de padecer sobrepeso, disminuye los niveles de estrés y ansiedad experimentado en las personas y 
brinda tiempo saludable de esparcimiento (Rodríguez, 2004 citado en Luci, 2009). Beneficios que pueden ser percibidos y experimentados desde el uso de las ciclovías, ya que estas como tal buscan la promoción del empleo de la bicicleta y otros medios de transporte activo (como son patines, patinetas y caminar) de manera segura con el fin de crear una movilidad más asequible, saludable y sostenible.

\section{Estrés, calidad de vida, y su asociación con el uso de ciclovías.}

Se considera importante antes de describir la potencial relación entre el uso del espacios publico urbanos como son la ciclovías con variables latentes como son calidad de vida y estrés, brindar una descripción de las definiciones de estrés y calidad de vida empleadas para justificar dicho proyecto así como su interacción con el modelo calidad de vida relacionado con la salud.

\section{Estrés Percibido/ Estado}

El estrés es una reacción psicofisiológica que permite que las personas puedan responder de manera activa a las demandas y condiciones del medio (Contrada, 2011). Mientras tanto, un estresor se define como aquella condición o evento que puede vincularse con cualquier componente de la vida que incide en la activación de una respuesta propia de estrés, donde el manejo de la activación de estas respuestas permite el declive de tensiones, además de permitir afrontar de forma eficiente y competente dichos estresores (Dorantes \& Matus, 2002).

Empíricamente se ha descubierto que el estrés es un factor que predispone la aparición de enfermedades y quebranto en la calidad de vida. Acorde con las conclusiones dadas por el Grupo de Trabajo Europeo de la Organización Mundial de la Salud se estimó que el estrés para el año 2020 podría llegar a convertirse en la principal causa de mortalidad asociado con la aparición de afecciones de carácter cardiovascular y trastornos depresivos ( Levi et al., 2001 citado en Schawartzmann, 2003).

Existen tres distintos enfoques sobre los cuales se basa la interpretación y abordaje del estrés, como es el enfoque que observa el estrés como una característica aversiva del ambiente, el enfoque que describe el estrés como un efecto fisiológico producto de los estímulos negativos y el enfoque que explica el estrés según la dinámica ambiente e individuo ( Leandro, 2012). Desprendiéndose de éste último enfoque el modelo propuesto por Lazarus y Folkman (1984), cual determina que el estrés psicológico o el estrés percibido debe ser visto como una relación particular entre el ambiente y la persona desde la perspectiva transaccional del estrés. Ésta perspectiva esboza que las 
personas responden de manera distintiva ante los estímulos estresores quedando sujeto a los estilos de afrontamiento e interpretaciones subjetivas con base a las características personales, experiencias e historia de las y los individuos (Lazarus \& Folkman, 1984).

El estrés estado en cambio, es la respuesta psicofisiológica-emocional momentánea activada ante la presencia de un estresor. Además, se ha encontrado que el estrés estado tiende a correlacionar de manera positiva con la ansiedad estado ( Alves, Cruz \& Aguiar, 2006). Acorde con el Organismo del Sistema Nacional de Salud de España (2009) se define ansiedad - estado como aquella que comprende un estado emocional transitorio que se caracteriza por presentar sentimientos subjetivos, conscientes, aprensivos e hiperactividad del sistema nervioso autónomo. Este tipo de ansiedad posee una fase transitoria y variable en cuanto a intensidad y duración, y es vivenciada por el individuo en un momento particular, además de reflejar síntomas como son aumento en la tensión muscular, dificultad de concentración y elevación de la tasa cardiaca (Organismo del Sistema Nacional de Salud Español, 2009).

El estrés y su medición han sido de interés tanto para la psicología como para otras ciencias de la salud. Se ha demostrado que los efectos derivados de la relación estrés y salud va a depender de la dinámica que se establezca entre carga genética y exposición a los diferentes factores ambientales (Mc Ewen, 2007 citado en Leandro, 2012). También se ha señalizado que las situaciones estresantes y la vivencia de niveles de estrés negativo se hallan vinculados con la etiología y fisiología de diferentes enfermedades tanto a nivel psicológico como físico (Cohen, Janick, Deverts \& Miller, 2007 citado en Leandro, 2012).

Altos niveles de estrés son un factor común que influye en el desarrollo de trastornos depresivos mayores, asociándose el inicio de la depresión con la presencia de situaciones cargadas de estrés; paralelamente la disminución de síntomas depresivos se vincula con la reducción en los niveles de estrés. Si la experiencia del estrés tiende a brindar respuestas más constantes y con mayor intensidad, eso incide en un deterioro en la calidad de vida (Farabaugh et al., 2004). A su vez Cardoso y Linnen (2012) explicitan que el estrés crónico interpersonal (presencia a largo plazo de dificultades y problemas permanentes dentro de las relaciones sociales) se vincula con la presencia de problemáticas en el área física y emocional.

En resumen, el estrés es un proceso integrado por diferentes dimensiones, entre ellas se pueden ubicar la dimensión percibida (estrés psicológico) y la dimensión estado, teniendo el entorno gran peso sobre la experiencia de estrés en sí.

\section{Calidad de Vida}

Antes de hablar sobre el concepto de calidad de vida es pertinente describir el Modelo Salutógenico, mismo que ha influido en la tendencia mundial referida 
a la promoción de la salud. Este modelo fue desarrollado por Antonsky (1979) y enfatiza sobre las condiciones y orígenes que permiten que una persona experimente bienestar y salud. Entre los conceptos que fundamente su teoría se encuentran:

Los recursos generales de resistencia, son los factores psicosociales, biológicos y materiales que permiten que una persona perciba su vida de modo coherente, comprensible y estructurada (Rivera, Ramos, Moreno \& García, 2011).

El Sentido de Coherencia, comprendido por la significatividad (ámbito emotivo), manejabilidad (ámbito instrumental) y la comprensibilidad (ámbito cognitivo) (Rivera et al., 2011).

Este modelo ha incidido en la implementación de la promoción de la salud, al basarse precisamente sobre el constructo de salud y no sobre el de enfermedad, ya que por medio de este enfoque se centraliza las acciones sobre la resolución de los conflictos, búsqueda de soluciones aunado con la identificación de recursos de resistencia propios de cada sujeto (Rivera et al., 2011). Características que también son tomadas en cuenta desde los modelos calidad de vida y calidad de vida relacionada con la salud (cvrs).

El concepto calidad de vida nace en la década de 1950 y es ampliamente difundido en 1980, siendo en primera instancia empleado dentro del campo de la medicina y posteriormente es tomado por la psicología y sociología quienes se encargan de vincular la calidad de vida con los constructos de felicidad y bienestar (Vinaccia, Japey, Martínez \& Arbelaez, 2008).

En sus inicios la calidad de vida se le asociaba netamente con el cuidado de la salud personal, conforme se fue avanzado en su elaboración teórica y estudiada a nivel empírico se le añade conceptualizaciones relacionadas con el campo de la salud e higiene pública, derechos laborales, derechos humanos, derechos ciudadanos, teniendo como base en cada uno de estos campos la preocupación y experiencia subjetiva de las personas ( Schawartzmann, 2003).

Desde el ámbito de la salud mental la calidad de vida es relacionada con orientaciones que promuevan mejoras en las condiciones de vida de las personas que presenten un trastorno psicológico o en aras de prevenir sus apariciones, para ello dicha relación ha sido explicada por diferentes enfoques como son el enfoque de satisfacción, funcionamiento del rol, el modelo calidad de vida propuesto por Schalock y el modelo combinado de importancia / satisfacción (Verdugo \& Marín, 2002).

El grupo Calidad de Vida de la Organización de Mundial de la Salud (World Health Organization Quality of Life, WHOQOL) (1995) esboza que la calidad de vida debe contemplar ejes a nivel subjetivo, multidimensional y funcionamiento tanto positivo como negativo. De acuerdo con el grupo el concepto calidad de vida hace referencia a la percepción individual sobre la 
posición en la vida dentro de su contexto cultural y sistema de valores con el cual interactúa, tomando en cuenta expectativas, metas e intereses.

El modelo calidad de vida relacionada con la salud se basa en el modelo calidad de vida, donde la principal diferencia entre dichos modelos radica en que los indicadores centralizados a evaluar la calidad de vida relacionada de la salud a nivel individual posee una mayor focalización y facilidad para que las personas logren centrarse en sí misma y valorar su salud, además de enfatizar en la unicidad de cada individuo para responder a la enfermedad y situaciones externas que atenten en contra de su bienestar.

La calidad de vida relacionada con la salud hasta la actualidad cuenta con diferentes conceptualizaciones provistas por distintos investigadores. Algunos la consideran como la consecuencia emocional y cognitiva del afrontamiento de estresores percibidos que atentan en contra de los objetivos o como la valoración subjetiva que las personas que presentan enfermedades dan sobre los ejes de su vida considerados importantes ( Urzúa, 2010). También es visualizada como aquel tipo evaluación subjetiva que incide en el estado de salud actual sobre la capacidad para obtener y mantener un nivel de funcionamiento general que colabore en la obtención de metas (Urzúa, 2010).

\section{Ciclovías y sus efectos sobre el estrés, calidad de vida}

En la actualidad el tema de movilidad en las ciudades es de suma importancia debido a la contaminación ambiental, densidad y congestión vehicular, poca accesibilidad a espacios públicos en conjunto con las inadecuadas formas de relación con la ciudad en sí. Según Velascos ( 2011) los patrones de movilidad centrados en la persona, la sostenibilidad y la ciudad, ayudan a que se revalore los espacios públicos, el sentido de pertenencia e identidad por parte de la ciudadanía con la ciudad.Entrando aquí en juego las ciclovías como espacios públicos constructores de movilidad y relación social.

De acuerdo con Mariano Rojas (2012) investigador del Centro de Investigación e Inteligencia Económica de Colombia, menciona que las sociedades progresan en tanto posean, promuevan y contribuyan al bienestar de sus ciudadanos y ciudadanas, asociado con la construcción de relaciones humanas y capital social, señalizando que el poder habitar activamente un entorno, influye en la calidad de vida así como en la salud mental.

Una investigación desarrollada en Colombia sobre la ciclovía en Bogotá, menciona que la ciclovía permite la apropiación del espacio público, además al gozar con normatividad legal y cultural favorece el establecimiento de pautas de interacción social (Gomescásseres, 2003).Para la evaluación de ésta ciclovía se efectuaron entrevistas semiestructuradas a diversos tipos de usuarios y usuarias, además de segmentar el sector en dos tramos, uno referido al alto potencial de uso 
y bajo potencial de uso. Se halla que en el sector con mayor frecuencia empleo, sus usuarios y usuarias recreaban mayores formas de interacción interpersonal y de autocuidado (Gomescásseres, 2003).

Otro estudio hecho en el año 2009 sobre la ciclovía en Bogotá que trabajó con una muestra de 1000 participantes (ciclistas) a los cuales se les aplicó la versión larga de la escala de Actividad Física Internacional junto a otras 10 preguntas centradas en explorar los constructos de confianza, eficacia, apoyo mutuo y componente social, concluye que el programa de ciclovía de esta ciudad ayuda en la creación de mejores espacios sociales, brindando mayor seguridad y bienestar general para sus usuarios y usuarias ( Sarmiento et al.,2010). Entre el análisis de datos empleados en dicha investigación se encuentran el uso de regresiones multivariadas con el objetivo de investigar la relación entre frecuencia de participación y niveles de capital social (Sarmiento et al., 2010).

A nivel de Costa Rica no se ha efectuado ningún estudio que permita valorar los efectos que las ciclovías pueden tener sobre los niveles de estrés y los índices de calidad de vida relacionada con la salud de sus usuarios y usuarias. Por ende se considera de interés la exploración de esta temática en Costa Rica con el fin de obtener datos que colaboren a reflejar de manera conceptual y empírica la existencia de posibles beneficios de índole psicológica relacionados con el uso de la ciclovía como catalizador de la actividad física y social.

\section{Discusión}

El espacio público urbano cuenta con un gran potencial de investigación desde el ámbito de las ciencias de la salud física y mental, siendo un importante centro de fusión en cuanto a beneficios psicológicos, físicos y sociales se refiere. Al realizar la revisión bibliográfica se halla que existe un creciente interés sobre la relación actividad física y bienestar psicológico pero se carece de estudios que investiguen de forma específica y rigurosa esta relación con el uso del espacio público urbano, en especial las ciclovías.

Las ciclovías son espacios públicos, interesantes a estudiar que pueden brindar datos de valor que permitan visualizar los efectos tanto positivos como negativos que tienen estos espacios urbanos a nivel objetivo y subjetivo sobre las personas que las emplean y comunidades en que se ubican; pudiendo ser un apoyo en la toma de decisiones comunitarias y gubernamentales asociadas a la creación de mayores áreas urbanas que prioricen un tránsito público, colectivo, activo y saludable.

Espacios públicos urbanos como son las ciclovías brindan condiciones que permiten el desarrollo de ciudades más sanas, lo cual influye de manera positiva sobre la percepción, abordaje y afrontamiento que tienen las personas ante estresores ambientales. A su vez dicho espacio, genera un mayor capital social, 
incentivando un aumento en los niveles de calidad de vida de sus habitantes, tal como lo señala Sarmiento y colaboradores (2010).

El esfuerzo ejecutado a la hora de pedalear al andar en bicicleta diariamente ayuda a mejorar la condición física, reduce el riesgo de sobrepeso, fortifica la estructura muscular, disminuye el estrés y ansiedad, además de brindar tiempo de esparcimiento (Rodríguez, 2004 citado en Luci, 2009).Consecuentemente, el uso de la bicicleta combate afecciones propias derivadas del sedentarismo como son la gestación de cardiopatías, enfermedades cardiovasculares e hipertensión ( Saraba, et als, 2005 citado en Luci, 2009). Situaciones que señalan los beneficios tanto a nivel físico como psicológico que puede tener el uso un transporte activo como es la bicicleta. Por tales motivos se considera de interés ahondar en la relación entre actividad física- uso de bicicleta y bienestar psicológico, relación que puede ser explorada desde el contexto público urbano como son los ciclovías, y brindar datos que problematicen sobre temas referidos a salud urbana y creación de ciudades saludables, sostenibles y accesibles.

Al promover el habitar activo de los entornos urbanos, no solamente se influye de manera positiva sobre la salud mental y calidad de vida de las personas sino que también contribuye sobre el progreso de las ciudades en sí. La implementación de la bicicleta como forma de movilidad activa además de favorecer la reducción de los gases de efecto invernadero, funciona como un medio para reactivar y recuperar el espacio público en conjunto al incremento de los niveles de cohesión social (Montoya \& Guzmán, 2013).

La calidad de vida al ser un constructo multidimensional y multidisciplinario debe ser abordada desde el ámbito ambiental, social, político, cultural e individual, por tanto el diseño urbano y espacio ambiental influye sobre los índices de calidad de vida de las poblaciones. Al implementarse la creación de ciudades saludables de manera directa se afecta la calidad de vida de las personas, ya que se les reivindica su derecho ciudadano de contar con un espacio urbano que promueve y proteja su medio tanto en el ámbito ambiental, mental, físico y social. Lo cual permite combatir la creciente aparición de síntomas depresivos, ansiosos, trastornos del sueño asociados a la contaminación atmosférica y acústica, así como el aumento en los niveles de obesidad y afecciones cormórbidas (cardiopatías, diabetes, embolias, cáncer de mama y colón) vinculadas a la falta de actividad física, factores que se hallan de forma intrínseca asociados a la dependencia que sea creado del transporte motorizado (ONU-Habitat, 2013).

Se encuentra que existe un creciente interés en el estudio de la relación actividad física y efectos psicológicos pero se carecen de investigaciones rigurosas que vinculen dicha relación dentro del contexto del espacio público urbano, y en menor medida si se trata de espacios específicos como son las ciclovías. Por ello con el fin de explorar la existencia de asociaciones directas entre espacio público urbano- bienestar psicológico y actividad física se recomienda que 
futuras líneas de investigación se estructuren desde diseños correlacionales que permitan reflejar dichas asociaciones. También se propone el abordar el estudio de las posibles relaciones entre uso de la bicicleta en espacios urbanos construidos como son las ciclovías y sus efectos sobre la ansiedad, depresión, auto-percepción y percepción urbana.

\section{Referencias}

ARQHYS. (2013). Ciclovías. Extraído el 6 de agosto del 2013 desde http://www. arahys.com/construccion/ciclovias.html

Alves, J.,Cruz, A., \& Aguiar, Z ( 2006). Adaptación para la lengua portuguesa de la Depressión, Axiety and Stress Scale (DASS). Rev Latino-Am Enfermagen, $14(6)$.

Antonsky, A. (1979). Health, stress and coping. New perspectives mental and physicall well-being. San Francisco: Jossey-Bass.

Augé, M. (2005). El no lugar y otras teorías. Extraído el 27 agosto del 2013 desde http://arellanos.blogspot.com/2005/06/marc-aug-el-no-lugar-y-otrasteoras.html

Cardoso, C., \& Linnen, A-M., (2012). Coping style moderates the effects on intranasal oxyitosin on the mood reponse to intepersonal stress. Experimental and a clinical psychopharmacology, 20 (2), 84-191.

Castro, J. (2005). Promoción de estilos de vida activa. Educación física y deporte, 24 (2), 49-64.

Castrogiovanni, A. (2007). Lugar, no lugar y entre: Los ángulos del espacio turístico. Estudios y perspectivas en turismo, 16, 5-25.

Contrada, R. J (2011). Stress, Adaptation and Health. The handbook of stress sciencie: Biology, psychology and health, 1-9.

Dorantes, C. \& Matus, G. (2002). El estrés y la ciudad. Revista del Centro de Investigación, 5 (18), 71-77.

Escobar, J. (2003). Actividad física como estilo de vida saludable: criterios básicos. Rev Med Risalda, 9 (2).

Farabaugh, A.,Mischoulon, D.,Fava, M., Green, C.,Gyyker, W.,\& Apert, J.(2004). La relación potencial entre niveles de estrés percibido y los subtipos de trastornos depresivos mayores (TDM). Revista de Toxicamanías, 4, 9-14. 
Ferrer, M. (2009). Re-creando el espacio público urbano. Política para construir ciudad y ciudadanía en Venezuela. Revista Ciencias Sociales, XV (1), 89-111.

Gomescásseres, T.( 2003). Deporte, juego y paseo dominical: Una aproximación de la ciclovía de Bogotá. Rev Colombiana de Sociología, 21, 175- 203.

Gómez, L., Mosquera, J., \& Jacoby, E. (2009). Salud, vida activa y ambiente urbanos en América Latina: Las ciudades vuelven a ser importantes en Velascos , N \& Rozowski, J. ( Eds), Obesidad ¿ qué podemos hacer? : Una mirada desde la salud pública. Santiago, Chile: Ediciones UC Pontifica Universidad Católica de Chile.

Guarino, L., Gavidia, I., Antor, M., \& Caballero, H. (2000). Estrés, salud mental y cambios inmunológicos en estudiantes universitarios. Psicología Conductual, $8(1), 57-71$.

Jacobs, M. (1992). The death and life of great American cities. New York: Vintage Books.

Jimenez, M., Miró., Martínez, P., \& Sánchez, A. (2008). Bienestar psicológico y hábitos saludables ¿Están asociados a la práctica de ejercicio físico? International Journal of Clinical Health Psychology, 8 (1), 185-202.

Lazurus, R., \& Folkman, S. (1984). Stress coping and adaptation. New York: Springer.

Leandro, M. (2012). Medición del estrés en seres humanos: Estado de la cuestión. Instituto de Investigaciones Psicológicas UCR. Costa Rica.

Low, S (2000). On the plaza. Politics of public space and culture. University of Texas, United States.

Luci, C. (2009). Ciclismo funcional ¿Promesa o quimera para la ciudad de Santiago, una respuesta a partir de los ciclistas funcionales y aquellos que no lo son? Tesis para optar por el grado de Magíster en Asentamientos Humanos y Medio Ambiente, Pontífica Universidad Católica de Chile, Santiago, Chile.

Marquéz, S. (1995). Beneficios psicológicos de la actividad física. Rev de Psicología. Gral y Aplic, 48 (1), 185-206.

Organismo del Sistema Nacional de Salud. (2009). Guía de práctica clínica sobre trastornos de la conducta alimentaria. Extraído el 17 octubre del 2012 desde http://www.guiasalud.es/egpc/conducta alimentaria/resumida/apartado05/ diagnostico 1.html 
Programa de las Naciones Unidas para los Asentamientos Humanos (ONUHabitat) (2013). Planificación y diseño de una movilidad urbana sostenible: Orientaciones para política. New York: Editorial Routledge.

Rivera, F., Ramos, P., Moreno, C., \& García, M. (2011). Análisis del modelo salutogénico en España: Aplicación en salud pública e implicaciones para el modelo activos en salud. Rev Esp Salud Pública, 85, 129-139.

Rojas, M. (2012). Hacia una sociedad con alta calidad de vida: Una propuesta de acción. Series Documentos Estratégicos, 4. Extraído el 23 de mayo del 2013 desde http://cecip.upaep.mx/investigacion/CIIE/assets/docs/doc00034.pdf

Schawartzmann, L (2003). Calidad de vida relacionada con la salud: Aspectos conceptuales. Ciencia y Enfermería, 10 (2), 9-21.

Tuesca, R. (2005). Calidad de vida, su importancia y como medirla. Rev Salud Uninorte, 21, 76-86.

Urzúa, A. (2010). Calidad de vida relacionada con la salud: Elementos conceptuales. Rev Med Chile, 138, 358-365.

Verdugo, M., \& Martín, M. (2002). Autoderminación y calidad de vida en salud mental: 2 conceptos emergentes. Salud Mental, 25 (4).

Velascos, A. (2011). Posibilidades de desarrollo urbano a partir de un sistema de transporte público en bicicleta. Extraído el 24 mayo del 2013 desde http:// movere.ec/documentos/desarrollourbnoyBP.pdf

Vinaccia, S., Japey, Q., Martínez, A., \& Arbelaez, C. (2008). Calidad de vida relacionada con la salud, emociones negativas y apoyo social en pacientes con psoriasis vulgar. Rev Psicología y Salud, 18 (01), 17-25.

WHOQOL Group. (1995). The World Health Organization Quality of Life Assement ( WHOQOL). Position paper from the World Health Organization. Soc Sci Med, 41, 1403-1409.

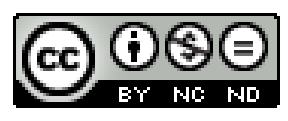

Este obra está bajo una licencia de Creative Commons ReconocimientoNoComercial-SinObraDerivada 4.0 Internacional. 